\title{
Les jumelages stratégiques au service de la compétition entre les villes
}

Strategic sister-state as service for cities competition

Strategische Städtepartnerschaften als probates Mittel im Standortwettbewerb

\section{Kuno Bucher et Nathalie Grillon}

\section{(2) OpenEdition}

\section{Journals}

Édition électronique

URL : http://journals.openedition.org/rge/1729

DOI : $10.4000 /$ rge. 1729

ISSN : $2108-6478$

Éditeur

Association des géographes de l'Est

Édition imprimée

Date de publication : 1 septembre 2008

ISSN : 0035-3213

\section{Référence électronique}

Kuno Bucher et Nathalie Grillon, « Les jumelages stratégiques au service de la compétition entre les villes », Revue Géographique de l'Est [En ligne], vol. 48 / 3-4 | 2008, mis en ligne le 02 mars 2010, consulté le 08 septembre 2020. URL : http://journals.openedition.org/rge/1729 ; DOI : https://doi.org/ $10.4000 /$ rge. 1729

Ce document a été généré automatiquement le 8 septembre 2020

Tous droits réservés 


\title{
Les jumelages stratégiques au service de la compétition entre les villes
}

\author{
Strategic sister-state as service for cities competition \\ Strategische Städtepartnerschaften als probates Mittel im Standortwettbewerb
}

Kuno Bucher et Nathalie Grillon

\section{NOTE DE L'AUTEUR}

Cet article s'appuie sur un mémoire réalisé par les auteurs dans le cadre du diplôme University Professional Stadt- und Regionalmanagement de l’Université de Bâle.

\section{Compétition régionale et mondialisation}

1 Le débat scientifique contemporain sur la mondialisation, qui se focalise sur les modèles d'interaction des dynamiques globales et locales, trouve son origine dans les études de Sassen (1991) relatives aux rôles des métropoles dans le capitalisme global. Contrairement à la perspective purement économique, la mondialisation portée par le concept de global city a pour la première fois tenu compte de multiples aspects sociaux. La compréhension moderne du concept de mondialisation tient en effet compte d'un ensemble de processus, qui modifient l'organisation spatiale des relations sociales ainsi que l'étendue géographique, l'intensité, la vitesse et l'efficacité des échanges. Il en résulte que de nouveaux courants décisionnels et de nouveaux réseaux d'action transcontinentaux ou interrégionaux apparaissent, principalement caractérisés par l'interaction de forces technologiques, politiques et économiques. L'influence de la distance spatiale diminue de plus en plus. 
2 Les paramètres de base qui ont permis cette évolution sont liés à l'ouverture politique et sociale envers les processus d'échange et à l'importance accrue de l'information dans la société. La compétitivité accrue entre les entreprises ne modifie pas seulement la marge de manœuvre des politiques menées par les firmes, mais influence en même temps de manière croissante les bases décisionnelles subjectives et le choix des cadres de vie individuels. Dans l'ambiance de la compétition relative aux facteurs de production immobiles, la main-d'œuvre a de plus en plus la possibilité de profiter de façon ciblée et systématique des différences offertes par les diverses localisations, ce qui lui permet de se déplacer vers les sites qui offrent les salaires les plus avantageux et la meilleure qualité de vie. Le choix de localisation individuel sera de plus en plus souvent le résultat d'une perception subjective de l'espace et dépendra ainsi fortement des représentations spatiales. Par conséquent, l'image des villes devient un élément de plus en plus central en matière d'information, d'évaluation et de décision; il échappe toujours plus aux acteurs politiques.

3 Dans le discours scientifique, le concept de réseau a fait de la ville une unité économique ou un niveau hiérarchique dominant. La focalisation sur les réseaux de villes a créé une nouvelle géographie des lieux centraux ou des lieux stratégiques qui permet d'analyser la mise en réseau spatiale comme une conséquence de la mondialisation. La mondialisation est entendue ici comme un phénomène social controversé, dépendant du contexte dans lequel il se développe et qui influence davantage les préférences des acteurs politiques qu'elle ne détermine les nouvelles structures sociétales de la politique mondiale.

4 Le débat sur la mondialisation a toutefois également montré que les formations politiques sont de plus en plus interdépendantes et que des acteurs non étatiques gagnent en importance dans l'arène politique. Cette évolution encourage les acteurs politiques - au niveau global ou local - à coopérer de manière générale et à élargir la palette des mesures stratégiques utilisées pour interagir avec un monde politique de plus en plus complexe. Dans ce sens, la mondialisation désigne la «mise en réseau transfrontalière croissante des espaces économiques et des activités du secteur privé, ce qui permet aux actions étatiques et aux intérêts privés de développer des modèles stimulant l'économie de marché " (Becker, 2007: 13 et suiv.) Dans un contexte de mondialisation des marchés, les gouvernements doivent faire adopter des mesures face aux groupes d'intérêt, qui tiennent compte des conditions sociales et dans l'intérêt de la sphère publique. L'augmentation de l'internationalisation de la politique conduit les politiques économiques à développer des accords de coopération avec d'autres Etats, dans l'espoir d'améliorer l'autonomie de la politique intérieure et la capacité à résoudre les problèmes relatifs à la politique économique.

5 L'institutionnalisation stratégique de ces relations d'échange ainsi que leur pilotage coopératif est fréquemment appelé gouvernance régionale (regional governance). Le changement du paradigme de gouvernement au paradigme de gouvernance exprime d'une part l'influence croissante des entreprises en matière de localisation économique et, d'autre part, le nombre et le poids politique croissant d'un certain nombre d'acteurs de la société civile.

6 Il est vrai que l'orientation prononcée en direction des réseaux a augmenté le potentiel de conflits entre acteurs jusqu'au niveau communal. Cependant, dans le même temps, cette évolution a permis de mettre en œuvre de nouvelles coopérations. En effet, on note que les collectivités locales cherchent de plus en plus souvent à s'associer de 
manière formelle ou informelle afin de faire valoir les intérêts de leurs communautés urbaines dans un contexte plus large qu'auparavant. La fragmentation du niveau étatique conjuguée à la mise en réseau de systèmes partiellement autonomes améliore ainsi de manière décisive la capacité à résoudre les problèmes, en comparaison avec des systèmes strictement hiérarchiques. Holm (2008) parle dans ce contexte d'upload governance.

7 Le renouveau régional aboutit de plus en plus souvent à une gestion stratégique désireuse de mettre en place des coopérations sous forme d'alliances stratégiques et d'ententes novatrices qui ressemblent à ce que construisent les entreprises mondialisées. Ces coopérations partiellement internationales ont pour propriété de s'établir de manière temporaire et de traiter de problèmes spécifiques. Elles ne reposent pas nécessairement sur des relations institutionnelles, mais sur des opportunités qui permettent d'exploiter de nouvelles ressources stratégiques. Afin d'atteindre une masse critique en matière de débouchés et de ressources, les centres urbains de rang comparable peuvent ainsi établir des coopérations ciblées. Cette approche est intéressante pour les villes qui sont confrontées aux défis de la compétition mondiale.

8 Les réseaux peuvent avoir des propriétés fonctionnelles diverses: les réseaux complémentaires mettent en œuvre une certaine division spatiale du travail grâce à des villes spécialisées (par exemple la Randstad Holland, la Vénétie, l'Emilie-Romagne), tandis que les réseaux de synergies se construisent entre partenaires ayant des spécialités comparables, formant un marché intégré et désireux de communiquer une image commune, par exemple en tant que centre financier multipolaire, cluster de recherche, ou région touristique. Finalement, les réseaux d'innovation travaillent à une innovation spatiale collective (technologie, infrastructure, etc.).

9 Si des avantages stratégiques découlent d'une participation en réseau, l'engagement et la flexibilité organisationnelle sont exigés en premier lieu. Les avantages à y participer augmentent avec l'augmentation de la durée et de l'intensité de la participation. La stratégie de comportement peut être opportuniste (maximisation du profit sans intention d'expansion, apport en ressources minimal), exploratoire (engagement important, processus d'apprentissage) ou stratégique. Cette dernière stratégie permet de mettre en œuvre un savoir ciblé, apporte une plus grande efficacité en termes d'informations, conduit à des réussites locales et à une ouverture vers la nouveauté. Des modèles de ce genre comportent également certains jumelages stratégiques, comme celui que Bâle-Ville a construit avec le Massachusetts ou avec Shanghai.

\section{La coopération dans la région trinationale du Rhin supérieur}

10 La région métropolitaine de Bâle est l'une des régions d'Europe les plus fragmentées du point de vue territorial. Depuis longtemps déjà, les frontières politiques ne correspondent plus au réseau complexe des imbrications économiques. Du fait de cette situation, il n'est donc pas étonnant que le canton de Bâle-Ville entretienne traditionnellement des relations multiples avec d'autres entités à un niveau national, bilatéral et multilatéral. L'échange institutionnalisé mis en œuvre dans le Rhin supérieur s'est ainsi développé sous forme de coopérations thématiques, comme le 
programme EUCOR établi il y a une vingtaine d'années entre les universités de Bâle, Fribourg-en-Brisgau, Karlsruhe, Strasbourg et Mulhouse.

Figure 1 : Carte de l'Eurodistrict Trinational de Bâle

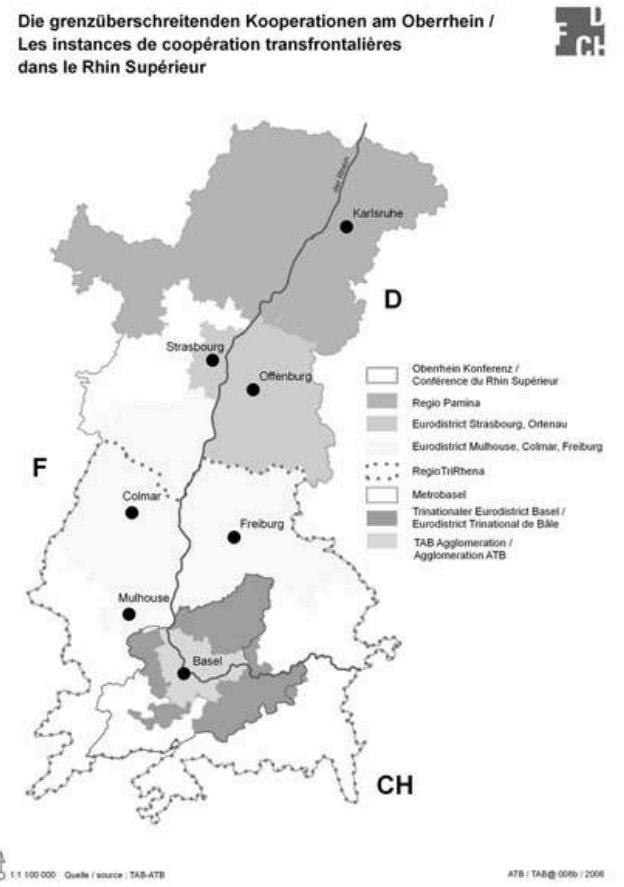

I'Eurodistrict qui porte en lui le processus d'intégration territoriale contribuera à l'amenuisement de la frontière entre les trois États.
A terme, c'est un véritable réseau urbain d'envergure métropolitaine qui pourrait voir le jour. En plus cette alliance territoriale renouvelle
les termes du dialogue entre la métropole báloise et ses voisines.

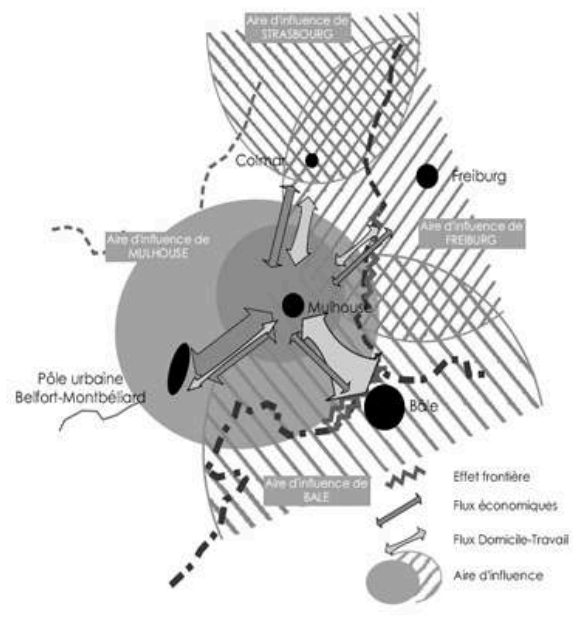

11 La dernière grande étape de la coopération régionale transfrontalière du Rhin supérieur est l'Eurodistrict Trinational de Bâle créé le 26 janvier 2007. Les thèmes prioritaires de cette coopération sont l'aboutissement des projets clés lancés dans le cadre du programme INTERREG III et l'achèvement du Concept de développement 2006-2020 de l'agglomération trinationale de Bâle, de même que l'aménagement du territoire, les transports publics et les services de santé (Chancellerie d'Etat du canton de Bâle-Ville, 2007 : 72).

Il a été très tôt reconnu que la ville de Bâle, en tant que centre urbain attractif et dynamique de petite taille à l'échelle de la région métropolitaine trinationale, était dépendante de la perméabilité des frontières étatiques et des coopérations qui pouvaient se nouer dans le cadre régional (Chancellerie d'Etat du canton de Bâle-Ville, 2007 : 40). L'impératif actuel demeure la mise en réseau des collectivités locales du Rhin supérieur et le positionnement collectif de l'agglomération trinationale sous le label bâlois.

13 L'effort consenti dans ce domaine est illustré par le nouveau département administratif (Präsidialdepartement) créé dans le cadre de la reforme du gouvernement de Bâle-Ville. Ce département réunit et revalorise les domaines des relations extérieures et de la promotion économique depuis janvier 2009. Ses missions principales consistent à mettre en œuvre des relations urbaines stratégiques ainsi que des activités à l'étranger. La politique extérieure de Bâle suit, dans ses grandes lignes, la stratégie de développement intitulée Basel 2020, qui décrit l'avenir de la ville. Dans ce document, Bâle est considérée comme le centre urbain de l'agglomération trinationale, dans lequel les habitants peuvent trouver des conditions d'habitation, de loisirs et de formation adaptées à leurs projets de vie les plus diversifiés. Certains facteurs clés de la ville 
devront être activement entretenus: spécialisation dans des activités fortement innovantes et dynamiques, formation et recherche de qualité, forte orientation internationale du marché du travail, existence de foires et de congrès attractifs, excellentes conditions économiques générales (législation du travail et fiscalité notamment), connections routières, ferroviaires et aériennes denses, attractivité culturelle et qualité de vie.

Les coopérations possèdent des caractéristiques spatiales et thématiques différentes et visent à renforcer l'attractivité résidentielle et professionnelle de Bâle dans un contexte compétitif caractérisé par une mobilité des facteurs de production. Le but de ces coopérations n'est pas uniquement de positionner à moyen ou à long terme Bâle au sommet du classement des villes, tant il est reconnu que les habitants doivent également s'épanouir dans un environnement harmonieux.

Le jumelage de villes et de collectivités locales peut prendre la forme de coopérations formelles et venir consolider la position des villes sur un globe mondialisé. En ce qui concerne Bâle, cette stratégie est relativement nouvelle en comparaison avec d'autres villes. Le partenariat intitulé Sister-State existant depuis 2002 entre Bâle-Ville et le Massachusetts aux Etats-Unis, ainsi que le jumelage formel signé en 2007 avec la métropole économique de Shanghai, située au sud de la Chine, peuvent être considérés comme les deux pierres angulaires de la politique extérieure de Bâle, aujourd'hui plus active et plus consciente de sa propre valeur. Afin de pouvoir mettre en œuvre cette politique avec succès, les structures régionales doivent travailler à renforcer les représentations individuelles de la région, désormais marquées par de nombreux niveaux administratifs et une forte fragmentation politique. L'objectif est alors de présenter la région comme un espace fonctionnellement intégré.

\section{Les jumelages au service de la politique extérieure municipale}

Bien que le terme de partenariat soit couramment employé, ce qualificatif issu des sciences politiques recouvre des domaines thématiques très divers, tels que le maintien de la paix, l'aide au développement ou le développement régional et urbain. A ceci s'ajoute le fait que les diverses formes de coopération intercommunales ont récemment connu un renouveau dans le cadre du débat autour de la gouvernance en réseau (network governance). De fait, les jumelages ne sont que des déclarations d'intention instituées entre deux collectivités locales, qui renforcent les contacts mutuels, y compris hors de la scène politique. Ces jumelages sont liés aux circonstances historiques de l'après Seconde Guerre mondiale, une période pendant laquelle les Etats ont cherché à renouer les échanges entre les populations et encourager le rapprochement des peuples. Ces jumelages sont souvent mis en œuvre par des initiatives personnelles de la part de personnes immigrées qui souhaitent maintenir un lien avec leur pays d'origine. Dans leur forme la plus moderne, les jumelages de villes peuvent être considérés comme des alliances permettant de renforcer les avantages économiques des différentes villes concernées et de construire des réseaux de coopération permettant de surmonter certains problèmes communs. A côté de cale, certains jumelages plus classiques demeurent, qui favorisent les échanges culturels ou l'aide au développement, souvent à l'initiative de citoyens. 

matière de politique extérieure, grâce à leur organisation en réseaux nationaux et internationaux, les acteurs territoriaux jouent également sur l'entretien de relations formelles à un niveau régional, bilatéral ou multilatéral pour mettre en œuvre une politique extérieure.

\section{L'exemple du Massachusetts}

Le canton de Bâle-Ville n'a opté pour une politique de jumelage active que très tardivement et a choisi la variante stratégique. Dans le rapport de stratégie de 2007 publié à la demande du Département de l'économie et des affaires sociales, le canton définit ces jumelages de la manière suivante: "Un jumelage stratégique est une coopération à long terme entre deux collectivités portant sur différents domaines, qui vise finalement aussi un renforcement durable des lieux concernés. A la base de la coopération, des accords réglés individuellement permettent d'optimiser le 
développement culturel, économique et politique avec la plus grande indépendance et flexibilité.» (Abt, 2007: 7). Jusqu'à la fin des années 1990, les jumelages au sens classique n'étaient pas des instruments au service de la politique extérieure de Bâle et toutes les demandes d'échanges envoyées aux services administratifs de la Chancellerie d'Etat recevaient une réponse négative. Ces réponses négatives étaient justifiées par le fait que l'entretien de relations privilégiées avec certaines villes ou certaines régions était de nature à repousser d'autres interlocuteurs potentiels. Le monde politique bâlois était convaincu (et l'est encore en partie actuellement) que la mise en place de jumelages particuliers conduirait à ce que d'autres portes se ferment ailleurs dans le monde.

La déclaration d'intention de développer et d'approfondir les relations dans les domaines du commerce, de l'économie, des sciences, de l'éducation, de l'art et de la culture, signée le 22 mai 2000 dans le cadre d'une visite de mission commerciale, forme la base du partenariat avec le Massachusetts. Certes, les déclarations d'intention sont une chose et la concrétisation en est une autre. Après la signature de la lettre d'intention (memorandum of understanding), le Département de l'économie et des affaires sociales du canton de Bâle-Ville a plusieurs fois exprimé son souhait de compléter la déclaration d'intention par une relation officielle, intitulée sister-state agreement (accord de jumelage) d'une plus grande valeur et permettant de valoriser davantage les contacts noués. Un tel accord constitue la relation politique la plus engageante qu'un Etat américain puisse conclure avec un autre Etat (au sens d'un canton suisse ou d'un Land allemand) étranger. Même si cet accord est un instrument politique de coopération de haute qualité, il n'inclut pas d'engagements juridiques ou financiers de la part des parties contractantes.

L'achèvement des négociations a eu lieu le 20 juin 2002 à Boston avec la ratification de la Proclamation of Sister-State Relationship between the Commonwealth of Massachusetts and the Canton of Basel-Stadt, Switzerland. Le Canton de Bâle-Ville et l'Etat du Massachusetts ont reconnu l'existence de similitudes: ils appartiennent aux pôles de croissance économiques de leur pays, se distinguent par une part importante d'entreprises hightech dynamiques, notamment dans le domaine des sciences de la vie, et disposent d'un environnement de recherche très pointu de même que d'instituts de formation renommés.

Un grand nombre de projets a pu être initié en sept années d'existence dans le cadre de cet accord : un échange scolaire régulier entre des lycées de Bâle et de l'agglomération de Boston, un échange d'expériences relatif aux projets de travaux publics dans le cadre du programme Urban Geosciences du Massachusetts Institute of Technology (MIT) et de l'Université de Bâle, une coopération de l'hôpital universitaire de Bâle avec le Beth Israel Hospital de Boston dans le domaine des soins infirmiers, un projet intitulé Drug Management entre la Harvard Medical School et l'hôpital universitaire de Bâle, un cursus de master eBusiness/eGovernment binational entre la grande école du nordouest de la Suisse et deux établissements d'enseignement supérieurs de Boston, ainsi que plusieurs travaux de recherche dans les domaines prometteurs des nanosciences et des biotechnologies. A cela s'ajoute le fait que la multinationale pharmaceutique Novartis exploite avec le Novartis Institute for BioMedical Research (NIBR) son infrastructure de recherche à Cambridge (MA) depuis 2002. Finalement, il existe bien entendu un échange entre plusieurs niveaux politiques de même qu'entre les organisations de promotion économique des deux partenaires. 
L'analyse stratégique des partenariats réalisés au cours des cinq premières années dans le cadre de l'accord entre Bâle-Ville et le Massachussetts a conduit à rédiger un dossier (portfolio) des partenaires impliqués, afin de pouvoir en déduire les champs d'action stratégiques prometteurs (voir Hess 2006). En ce qui concerne Bâle-Ville, les critères de succès ainsi que les mesures à prendre s'accordent avec les objectifs du document stratégique Basel 2020. Ils s'orientent fortement sur le renforcement des clusters stratégiques dans le domaine de l'économie et de la recherche. Concernant le jumelage avec le Massachusetts, la stratégie de coopération menée dans le domaine des sciences et de la formation est confirmée par l'analyse des portfolios. Les résultats montrent que Bâle est encore loin d'avoir atteint les objectifs visés et qu'en absence de mesures internes permettant d'accroître l'envergure de la coopération, le canton risque de ne poursuivre que des coopérations ponctuelles dans lesquelles l'engagement stratégique n'est plus rentable. En particulier, cette analyse montre que certains potentiels latents existent, qu'il s'agit de développer en identifiant précisément les forces et les faiblesses du partenariat.

Figure 2 : Portfolio du partenariat entre Bâle et le Massachusetts

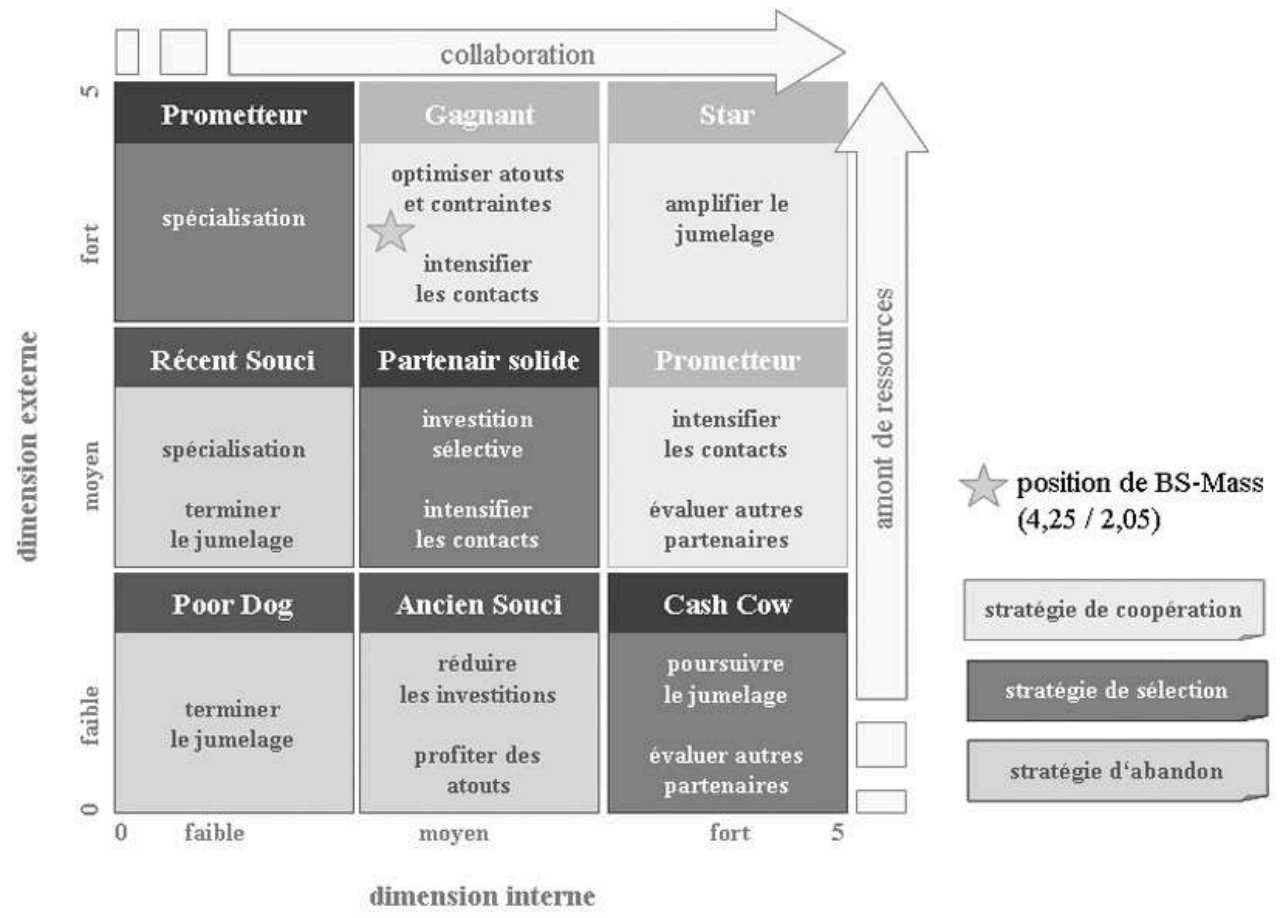

L'évaluation a identifié le maillon faible de la relation entre Bâle et le Massachusetts : il s'agit du réseau de contacts et de la structure organisationnelle, qui sont insuffisamment développés, malgré la création de l'association Friends of Massachussetts, Basel, créée pour y remédier. Le lancement d'activités successives nécessite de renforcer la structure organisationnelle, ce qui pourrait être facilement achevé compte tenu des conditions générales existantes dans les deux Etats. Le portfolio relatif au Massachusetts montre en outre que Bâle pourrait soutenir l'élargissement des coopérations du côté américain, de manière à profiter des compétences de l'Etat américain.

La plate-forme swissnex Boston atteste que les domaines de l'éducation et de la recherche forment deux champs d'activités importants. L'intensification des contacts 
personnels avec la représentation locale de swissnex (aussi bien à Boston qu'à Shanghai) pourrait permettre de favoriser la réalisation de projets communs. L'analyse montre que la poursuite du partenariat devrait tenir compte des éléments suivants :

- Les relations personnelles et institutionnelles existantes sont indispensables pour permettre la réactivation du partenariat et sont à considérer comme un investissement à long terme.

31 - Il existe déjà un réseau informel dense dans les domaines de l'éducation et de la recherche à partir duquel les relations pourraient être approfondies.

32 - Le cluster des sciences de la vie de Bâle constitue un point d'attraction pour la recherche et l'économie.

33 - Les domaines du développement durable et de l'énergie (green/clean tech) possèdent un important potentiel d'avenir.

34 - Le partenariat avec la région de Bâle peut être considéré comme une porte d'entrée vers l'Europe.

- Les contacts de Bâle avec la Chine (Shanghai) sont un atout supplémentaire important.

Le partenariat avec le Massachusetts est jugé de façon relativement critique du côté de Bâle. La mise en œuvre des projets réalisés jusqu'à présent est perçue comme ayant eu peu de succès. La faible popularité du jumelage est souvent attribuée à un manque de programme concret et à un engagement en partie déficient - également du côté du gouvernement. La région de Boston/Massachusetts est généralement considérée comme un partenaire approprié même si un partenariat avec Boston ou Cambridge aurait peut-être été plus tangible. Cependant, les observateurs notent qu'une prolongation des coopérations est souhaitable, notamment du point de vue de la recherche (sciences de la vie et médecine) et de la promotion économique.

37 Outre les aspects organisationnels et liés aux réseaux déjà décrits, une enquête effectuée auprès de certains acteurs a permis de montrer que la phase de départ du partenariat avait été difficile. Même si l'accord est ciblé principalement sur une coopération économique ayant un objectif stratégique, certains points fondamentaux ainsi qu'un programme de mise en œuvre des coopérations manquaient dès le départ. Ces éléments auraient permis de prévoir des objectifs à atteindre et des délais à respecter.

38 Il convient de retenir qu'un jumelage de villes aura probablement toujours un caractère idéel, dans la mesure où il s'agit finalement d'un processus d'échange sur un plan politique, économique et culturel, dont la valeur ne se manifeste qu'indirectement en monnaie sonnante et trébuchante. Néanmoins, ces jumelages doivent être considérés comme des investissements à long terme qui vivent principalement d'expériences positives réalisées à court terme. Un jumelage nécessite ainsi beaucoup d'énergie et d'engagement, il doit être visible pour la population et pour toutes les personnes impliquées. La relation doit s'ancrer dans une stratégie plus large et s'accorder avec le concept général de la politique extérieure de Bâle-Ville.

Le renouveau de contacts bilatéraux, si possible lié à des projets concrets, est envisageable dans le cas du jumelage entre Bâle-Ville et le Massachusetts. Malheureusement, les activités de réseautage (networking) sont entravées du côté américain par la forte mobilité professionnelle des personnes de référence, qui changent de fonction ou d'emploi après deux ou trois ans et cessent leurs activités dans 
le réseau. L'exemple le plus récent est fourni par la démission de la directrice du Massachusetts Office of International Trade \& Investment, Christa Bleyleben, avec qui une relation étroite existait depuis le début du jumelage. Malgré ces changements, il est important que les relations personnelles continuent à exister indépendamment de l'occupation actuelle des personnes dans le réseau. La figure suivante identifie les acteurs centraux du cluster Education-Recherche-Sciences de la vie. Elle indique aussi bien les ponts existants que les éventuelles relations qui pourraient être mises en œuvre dans le futur et sur lesquelles reposent la réalisation du projet.

Figure 3 : Cluster recherche - Life Sciences Bâle - Massachusetts

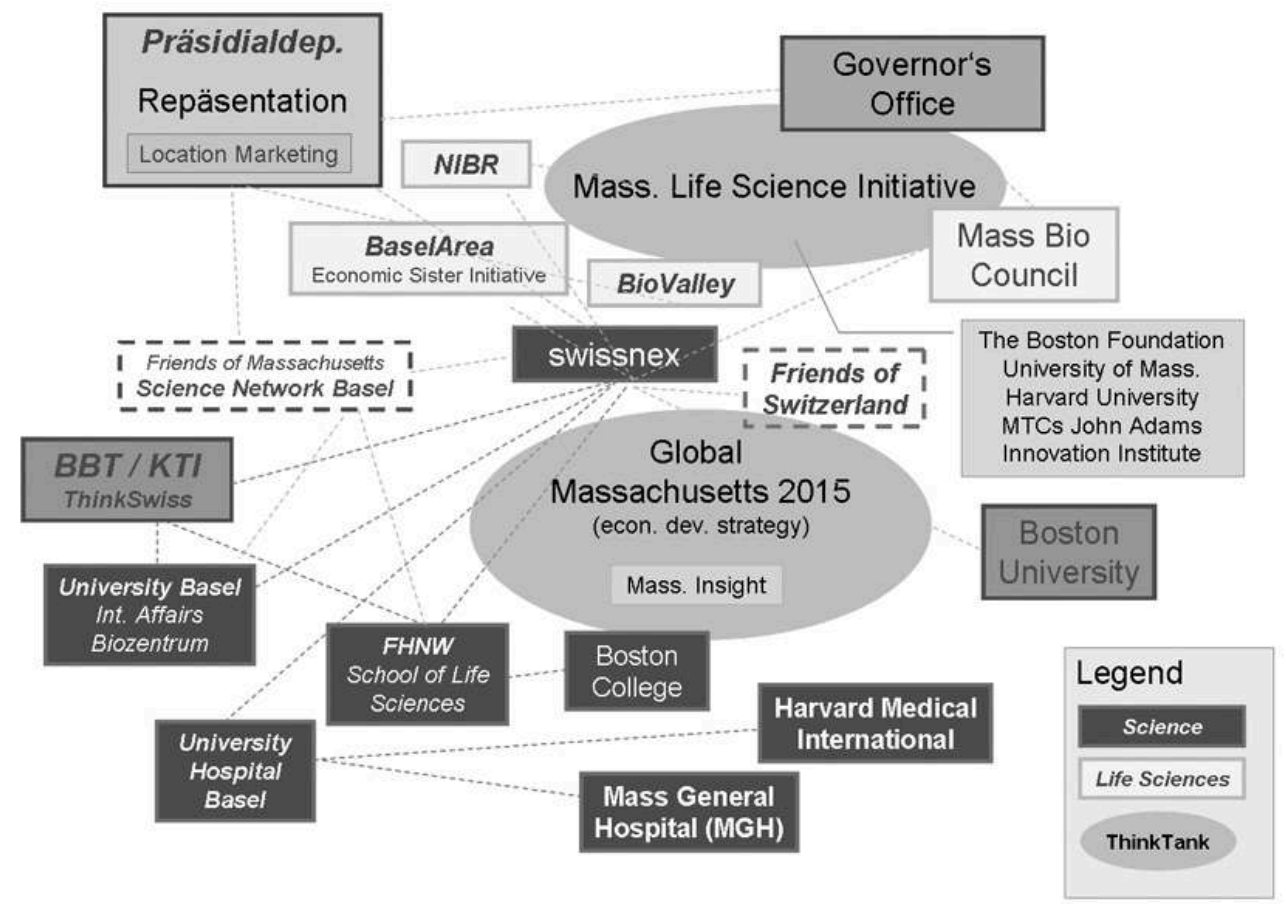

Au mois de mai 2009, le partenariat formel entre Bâle-Ville et le Massachusetts aura neuf ans. Que s'est-il passé durant ces années ? Peu de choses, comparativement à ce que ce que le monde politique et l'association Friends of Massachussetts avaient espéré lors de la ratification. Malgré ce constat, il est possible de renouveler les collaborations sur les bases existantes et notamment de focaliser les activités dans les domaines des sciences et de l'éducation, qui ont montré les résultats les plus tangibles jusqu'ici. Le réseau de contact actuel devrait se transformer en plate-forme d'échange ouverte dans le domaine de la recherche et de la formation, sous le nom de Science Network Basel.

\section{Le partenariat avec le Massachusetts et la compétition des régions}

41 Avec la formalisation du jumelage entre Bâle et Shanghai, les jumelages stratégiques entre collectivités sont devenus une partie intégrante de la promotion du site bâlois et un élément de la compétition que se livrent les régions à l'échelle mondiale. Les jumelages sont intégrés dans la stratégie cantonale générale en tant que mesure permettant de renforcer les atouts existants (Université de Bâle, 1998) et sont 
considérés comme un élément important du réseau de relations internationales de Bâle-Ville. Le canton focalise son attention sur les activités ayant des répercussions positives directes ou indirectes sur le développement de la région et sur les objectifs centraux formulés dans le document Basel 2020. A cet égard, le jumelage avec le Massachusetts devrait contribuer au renforcement des activités de recherches conduites dans la région, apporter une plus-value appréciable pour Bâle et renforcer la visibilité ainsi que la position de Bâle dans la compétition internationale qui oppose les centres de formation et de recherche.

Le jumelage se prête idéalement à l'objectif de développer davantage Bâle comme un centre de formation et de recherche de haut niveau. Le potentiel de synergie est important, considérant les 70 établissements de formation supérieurs présents dans la région de Boston, y compris les hôpitaux universitaires les plus connus des Etats-Unis. Mais Bâle a également des atouts importants à faire valoir, notamment l'un des hôpitaux universitaires les plus reconnus de Suisse, la plus ancienne université du pays avec ses deux domaines prioritaires que sont les sciences de la vie et la culture, la Haute école du nord-ouest de la Suisse, le Department of Biosystems Science and Engineering de l'Ecole polytechnique fédérale de Zurich, l'ETH Studio Basel actif dans l'architecture ainsi que de nombreux laboratoires de recherche travaillant pour l'industrie pharmaceutique et les biotechnologies. La stratégie 2007 de l'Université de Bâle a déjà permis de franchir un premier pas dans la direction souhaitée. Cette stratégie affirme que: «L'Université de Bâle offre toutes les facultés et se distingue par son offre pédagogique diversifiée mais clairement définie. Elle revendique cette diversité en ayant conscience de ses limites, en recherchant les possibilités de mise en réseau nationales et internationales et les coopérations, tout en les mettant en œuvre systématiquement. [...] Dans la recherche, la qualité est soutenue dans tous les domaines du savoir par une visibilité internationale » (Université de Bâle, 2007).

La stratégie de coopération dans le domaine de la formation et des sciences suit quatre orientations stratégiques : (1) Bâle s'engage à jouer un rôle actif et à prendre l'initiative lors de la réalisation de projets communs orientés selon la stratégie 2007 de l'Université de Bâle. Cinq centres de compétences interdisciplinaires devraient être créés sous le nom de Basel Network of Excellence in Life Sciences. Du point de vue thématique, ces centres contribuent parfaitement au cluster de formation et de recherche et font de Bâle un partenaire attractif pour des collaborations interuniversitaires. (2) Le réseau de formation établi entre Bâle et le Massachusetts se concentre sur des activités exerçant une influence positive directe ou indirecte sur la réalisation de la stratégie de Basel 2020. Ces activités renforcent ainsi la position de Bâle dans la compétition des régions au plan mondial. Le réseau relationnel de la Haute école du nord-ouest de la Suisse devrait contribuer à une utilisation des potentiels de synergie dans le domaine de la santé et de l'économie (développement commercial, startups). (3) Le réseau de partenaires est conçu comme une plate-forme de communication et d'information qui implique les acteurs des deux sites et qui renforce et rend plus visible les avantages de Bâle. L'utilisation des technologies de communication modernes facilite la mise en réseau des acteurs et encourage l'échange entre des réseaux individuels de personnes (par exemple des groupes d'intérêt ou des réseaux sociaux). (4) Les activités réalisées dans le cadre du partenariat reposent sur le modèle du partenariat public-privé et l'inclusion des acteurs concernés. Les ressources publiques sont concentrées sur des 
projets centraux qui renforcent durablement les relations entre la région de Bâle et le Massachusetts et qui sont perceptibles par le grand public.

Certains événements annuels marquants pourraient être organisés alternativement à Bâle et au Massachusetts. La série d'événements pourrait être lancée par exemple par une journée (une semaine ou un mois) officielle de partenariat. Dans le domaine de la formation de base, les programmes d'échanges d'élèves et d'enseignants existants pourraient être approfondis et poursuivis au niveau des études supérieures. Bâle, en tant que ville de foires et de congrès située au centre de l'Europe, pourrait mettre en œuvre des liens privilégiés entre les innovations et la production de niche hautement spécialisée, dans le cadre de son cluster des sciences de la vie.

Le point de départ d'une coopération approfondie pourrait être la formation de groupes de travail dans les domaines des nanotechnologies (entre le MIT et l'Université de Bâle), de la médecine ou des soins hospitaliers. La commémoration du $550^{\text {ème }}$ anniversaire de l'Université de Bâle pourrait jouer un rôle important pour réunir ces énergies, d'autant que les universités américaines attachent généralement une grande attention aux traditions de ce genre. Cet événement permettrait de lancer des partenariats ciblés ou d'inviter des professeurs de renommée internationale. Un avantage indiscutable de cette démarche pragmatique est d'utiliser des plateformes existantes, ce qui permet de transférer des connaissances et de gagner en réputation et en visibilité internationale sans toutefois nécessiter des dépenses trop importantes. Afin d'accroître la visibilité de Bâle auprès des nombreuses universités et hautes écoles de Boston, la plate-forme swissnex Boston, bien introduite dans le monde académique, constitue un excellent point d'ancrage permettant de construire et d'étendre des réseaux de partenaires. A ceci s'ajoute le fait que les régions engagées dans la démarche de coopération bénéficient des programmes de promotion de la recherche, tels que ThinkSwiss, qui leur permettent de se positionner sur la scène internationale.

Figure 4 : Carte du réseau swissnex

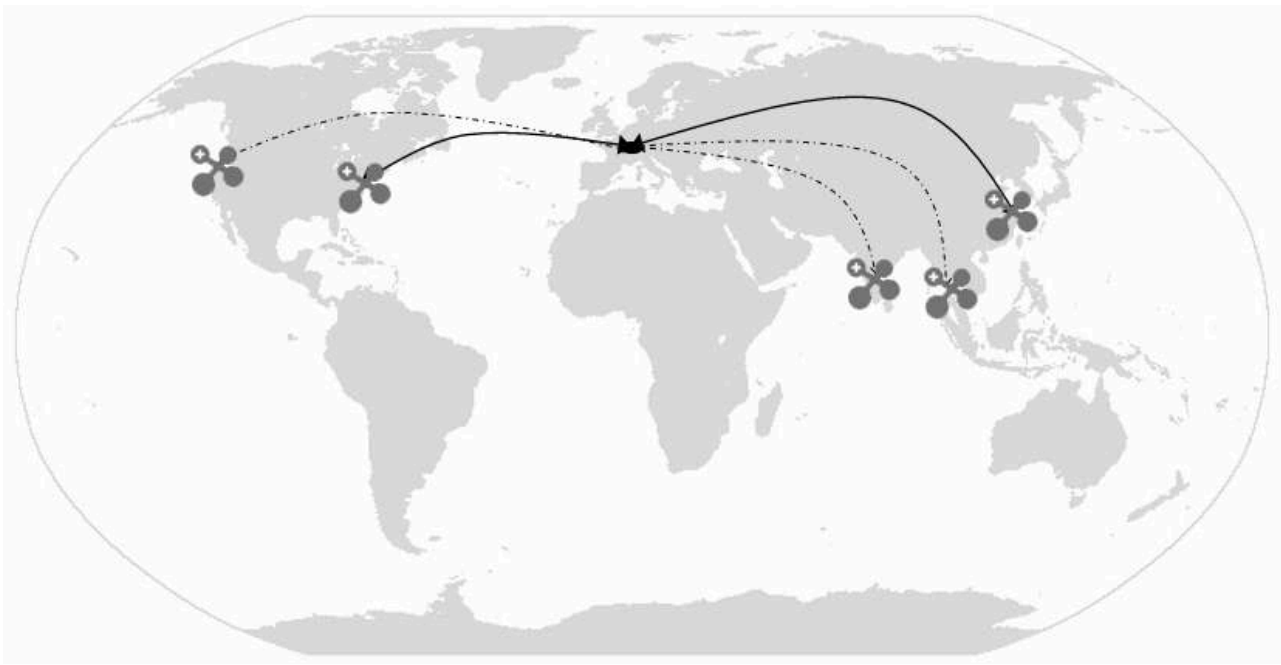




\section{BIBLIOGRAPHIE}

Abt M., Behofsits S., Horvath S., Mazzei C., Tivolino R. (2007). - Basel meets Shanghai. Planung und Umsetzung seiner strategischen Städtepartnerschaft, aufgezeigt am Beispiel Basel-Shanghai, Lugano, Université de la Suisse italienne.

Becker, M., John S., Schirm S. (dirs) (2007). - Globalisierung und Global Governance, Paderborn, Utb Wilhelm Fink.

Commission européenne (2007). - Programme «L'Europe pour les Citoyens » 2007-2013, http:// eacea.ec.europa.eu/citizenship/guide/documents/programme_guide_fr.pdf (accédé le 30 juin 2009).

Hess M. (2006). - Modell zur Auswahl, Bildung und Festigung transnationaler Partnerschaften als Instrument der Netzwerkorientierung im Standortmarketing. Bewertung der Partnerschaft BaselMassachusetts und Ableitung von Handelsimplikationen für die Partnerschaft Basel - Shanghai, Université de Bâle.

Holm A. (2008). - « Privatisierung der kommunalen Wohnungsbestände », in Gestring N., Glasauer H., Hannemann C. (dir.). - Jahrbuch StadtRegion 2006/2007 Schwerpunkt : Arme reiche Stadt, Leverkusen, Opladen, Budrich, p.101-109.

Sassen S. (1991). - The Global City, New York, London, Tokyo, Princeton, Princeton University Press.

Chancellerie d'Etat du canton de Bâle-Ville (2007). - Politikplan 2008-2011. Das Planungs- und Steuerungsinstrument des Regierungsrates mit Bilanz 2006/2007, Bâle, Basler Druck + Verlag AG.

Université de Bâle (2007). - Die Strategie 2007. Zur Entwicklung der Universität Basel 2007-2013, Bâle, Steudler Press.

Université de Bâle (1998). - 10 Stärken von Basel. Beiträge der Universität Basel für die Werkstadt Basel, Bâle, Stelle für Öffentlichkeitsarbeit, Université de Bâle.

\section{RÉSUMÉS}

La mondialisation réduit généralement les possibilités d'organisation politique ainsi que la marge de manœuvre des collectivités locales en termes de décisions politiques. Les acteurs politiques essaient de faire face à ces nouveaux défis à travers des modèles d'action alternatifs et des formes organisationnelles adaptées. Les approches et concepts proposés dans ce travail forment la base d'idées permettant l'encadrement pratique d'une stratégie de politique extérieure de Bâle. L'article introduit brièvement les changements ayant eu lieu en matière de compétition entre les régions ainsi que les nouveaux défis de la gestion urbaine et de la politique locale. Il commente ensuite de manière plus approfondie les champs d'action actuels de la politique extérieure du canton de Bâle-Ville dans l'espace de l'agglomération trinationale. Pour finir, en s'appuyant sur l'exemple du partenariat de Bâle avec le Massachussetts, l'article examine le rôle des coopérations dans l'élaboration de la stratégie de développement de Bâle 2020.

Economic globalisation changed the scope of decision making and politicising for local municipalities. Political players seek to face these new challenges with alternative action and organisational patterns. This article illustrates the core approaches and concepts that build the theoretical foundation to reduce this 'external Basel-strategy' to practice. The short preliminary 
outline of altered conditions in strategic location marketing is followed by an overview on the current domains of foreign policy in the trinational Basel metropolitan area. The concluding showcase of the Sister-State relation between the Canton of Basel-Stadt and the Commonwealth of Massachusetts demonstrates that co-operations can be a strategic element in implementing the local development strategy Basel 2020.

Im Zuge der ökonomischen Globalisierung verringern sich die Gestaltbarkeit der Politik und der Entscheidungsspielraum örtlich agierender Gebietskörperschaften. Die politischen Akteure versuchen, diesen neuen Herausforderungen mit alternativen Handlungsmustern und massgeschneiderten Organisationsformen $\mathrm{zu}$ begegnen. Die im Folgenden exemplarisch erläuterten Ansätze und Konzepte bilden das gedankliche Grundgerüst für die praktische Einbettung einer 'aussenpolitischen Basel-Strategie'. Einleitend wird ein kurzer Abriss über die durch den regionalen Standortwettbewerb veränderten Rahmenbedingungen und neuen Herausforderungen für Stadtmanagement und Lokalpolitik präsentiert, bevor die aktuellen aussenpolitischen Handlungsfelder des Basler Stadtkantons im trinationalen Agglomerationsraum näher erläutert werden. Abschliessend soll am Beispiel der Staatspartnerschaft mit dem US-Bundesstaat Massachusetts gezeigt werden, welche Rolle Kooperationen als strategischer Baustein auf dem Weg zur Entwicklungsstrategie Basel 2020 spielen.

\section{INDEX}

Mots-clés : Bâle, cluster de recherche, compétition des régions, coopération, jumelage, Massachusetts, mondialisation, politique extérieure municipale, promotion économique Schlüsselwörter : Basel, Forschungscluster, Globalisierung, kommunale Aussenpolitik, Kooperation, Massachusetts, Städtepartnerschaft, Standortwettbewerb, strategisches Standortmanagement

Keywords : Basel, cooperation, globalization, local foreign policy, Massachusetts, regional competition, research cluster, sister-state, strategic location marketing

\section{AUTEURS}

\section{KUNO BUCHER}

Service de la statistique du canton de Bâle-Ville - Binningerstrasse 6, CH-4001 Bâle kuno.bucher@bs.ch

\section{NATHALIE GRILLON}

Service de la statistique du canton de Bâle-Ville - Binningerstrasse 6, CH-4001 Bâle natalie.grillon@bs.ch 